\title{
Port-Hamiltonian Modeling for Soft-Finger Manipulation
}

\author{
F. Ficuciello, R. Carloni, L.C. Visser and S. Stramigioli
}

\begin{abstract}
In this paper, we present a port-Hamiltonian model of a multi-fingered robotic hand, with soft-pads, while grasping and manipulating an object. The algebraic constraints of the interconnected systems are represented by a geometric object, called Dirac structure. This provides a powerful way to describe the non-contact to contact transition and contact viscoelasticity, by using the concepts of energy flows and power preserving interconnections. Using the port based model, an Intrinsically Passive Controller (IPC) is used to control the internal forces. Simulation results validate the model and demonstrate the effectiveness of the port-based approach.
\end{abstract}

\section{INTRODUCTION}

Dextrous manipulation skills, for personal and service robots in unstructured environments, are of fundamental importance in performing different tasks. Usually, a robotic hand has to manipulate objects of different shape, size, weight, material, and, in some cases, has to interact with human beings. During manipulation, the dynamic properties of the controlled system change, due to the non-contact to contact transitions and due to the contact viscoelasticity. Therefore, in order to derive the dynamic model of a handobject system during grasping, the contact model between the fingers and the object is of crucial interest.

In the Lagrangian formulation, the dynamic model of the hand-object system takes the form of a multibody system. In case of rigid contact, the whole system is a nonholonomic constrained system and the equations can be obtained using the Lagrange-D'Alembert formulation, considering the grasping constraint equations [1]. In case of a compliant contact model, where the fingers have thick compliant layers of viscoelastic material, the grasping constraint equations are not valid anymore. Moreover, the dynamics of the contact are influencing the system dynamics, and have to be considered.

The port-Hamiltonian framework is based on describing a system in terms of energy variables and the interconnection of systems by means of power ports. Any physical system can be described by a set of elements storing kinetic or potential energy, a set of energy dissipating elements, and a set of power preserving ports, through which energy can only be transferred and not produced [2]. The energy flow

\footnotetext{
This work has been partially funded by the European Commission's Seventh Framework Programme as part of the project DEXMART under the grant agreement ICT-216239. This work has also been partially funded by The Netherlands Ministry of Economic Affairs as part of FALCON project under the responsibility of the Embedded Systems Institute (BSIK03021) program with Vanderlande Industries as the industrial partner.

f.ficuciello@unina.it, Dipartimento di Informatica e Sistemistica, Università degli Studi di Napoli Federico II, Italy. \{r.carloni,l.c.visser,s.stramigioli\}@utwente.nl, Department of Electrical Engineering, Faculty of Electrical Engineering, Mathematics and Computer Science, University of Twente, The Netherlands.
}

variables are intrinsically defined and are independent of the particular configuration of the physical systems. The concept of a power port is an efficient and useful way to describe the interaction between physical systems and between the system and the environment.

The theory of port-Hamiltonian systems allows to describe the system behavior in a coordinate-free way and can be naturally extended to include constrained systems and compliant contact models. This approach is useful to model and control the interaction between a robot and a passive environment. The robot is a $n$-DOF mechanical passive system with respect to the controller, that can be modeled as a port-Hamiltonian system. To preserve a passive behavior in the interaction with the environment, both in case of contact and non-contact, an Intrinsically Passive Controller (IPC) [3], based on impedance control [4], can be used.

Since the IPC approach yields an intrinsically passive system, the controlled system will be stable, both in case of contact and non-contact phases, for every passive, even unknown, environment. This is in contrast with a conventional hybrid controller, which switches from position to force control when a contact occurs. Such controllers can easily become unstable, because of noise affecting the force sensors that detect the contact. Moreover, a hybrid forceposition control requires a perfect planning of the tasks, which is only possible if the environment is known.

In this paper, we present a port-Hamiltonian model of a multi-fingered robotic hand, with soft-pads on the finger tips, grasping an object. The viscoelastic behavior of the contact is described in terms of energy storage and dissipation. Using the concept of power ports, the dynamics of the hand, the contact, and the object are described in a coordinate-free way. Moreover, an IPC is applied to control the motion of the object and to regulate the internal forces, i.e. the forces applied at the contact points and not influencing the object motion. These forces are important to have a stable grasp. In the model of the hand-object system, we assume that the contact forces are always satisfying the friction cone conditions, i.e. the contact forces are always inside the friction cone, and we assume that there is no rolling contact. This means that the grasp matrix is constant.

The main advantage of the port-Hamiltonian formulation for constrained systems is that we do not need to modify the dynamic equations when a change occurs in the contact state. Instead, it is possible to represent both cases in a time-dependent geometrical structure, that satisfies the power continuity conditions in every contact state. This framework allows to approach the problem in a more intuitive and compact way. The graphic bond graph representation of 
the system is based on the energy flow through the ports connecting the single components.

The paper is organized as follows. In Sec. II, we formulate the problem in a port-based setting and present the mathematical framework. In Sec. III, we derive the dynamics of the contact model. In Sec. IV, we propose the port-Hamiltonian model of the system, based on the analysis of the energetic interconnections and the properties of the subsystems. In Sec. V, simulation results are presented to validate the model and to show the behavior of the system when an IPC based controller is applied. Finally, Sec. VI provides conclusions and sketches the future work.

\section{PORT-BASED FORMULATION}

The port-Hamiltonian formalism has been introduced by van der Schaft and Maschke in [6]. Port-based modeling is at the basis of network theory, in which the different parts of the system are interconnected through power ports and described in terms of power exchange. A power port is defined by a pair of dual variables, a flow $f$ and an effort $e$, whose intrinsic dual product $\langle e \mid f\rangle$ yields power. If $\mathcal{V}$ is the linear space of flows, then the dual space $\mathcal{V}^{*}$ is the linear space of efforts. On the space $\mathcal{V} \times \mathcal{V}^{*}$, it is possible to define a power continuous structure, called Dirac structure, which defines the interconnection between the power ports, i.e. it describes how the power is distributed between the ports. The Dirac structure is a subspace $\mathcal{D} \subset \mathcal{V} \times \mathcal{V}^{*}$ such that [7]:

$$
\mathcal{D}=\left\{(f, e) \mid\langle e \mid f\rangle=0, \quad \forall(f, e) \in \mathcal{D} \subset \mathcal{V} \times \mathcal{V}^{*}\right\}
$$

A generic Dirac structure is depicted in Fig. 1. The bonds, connected to the structure, realize the ports through which energy can be exchanged with energy storage elements, energy dissipating elements, the controller, and the interaction port, with which the system interacts with the environment. In order to derive the mathematical model of the manipulation system as a port-Hamiltonian system, we need to define a state manifold $\mathcal{S}$, of which the coordinates represent energy variables, and on $\mathcal{S}$ a Hamiltonian energy function $H: \mathcal{S} \rightarrow \mathbb{R}$ describing the total energy of the system. Then, by making explicit the Dirac structure, the system dynamics can be derived. Since an interconnection of portHamiltonian systems is again a port-Hamiltonian system, we can proceed by individually modeling the hand, the object that is manipulated, and the contacts, and to define the interconnection of the systems.

Regarding the Dirac structure of the contact model, observe that the contact represents the power continuous interconnection between the finger, the soft-pad and the object, in terms of elastic energy storage and energy dissipation. This interconnection can be represented considering the finger and the object as two rigid bodies connected trough a viscoelastic soft-pad. Since in a manipulation task both contact and non-contact situations may occur, the Dirac structure is not constant in time [8]. The contact and non-contact state are both represented in the same switching Dirac structure, which is, obviously, time dependent.

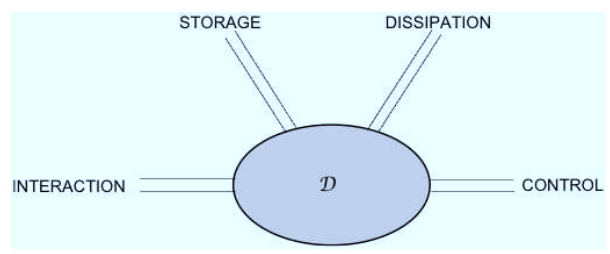

Fig. 1. Dirac structure of a generic port-Hamiltonian system.

\section{A. Problem statement}

In the Lagrangian formulation, the dynamic model of a $n$-fingered hand, each with $r$ degrees of freedom, has the form:

$$
\mathbf{M}(\mathbf{q}) \ddot{\mathbf{q}}+\mathbf{C}(\mathbf{q}, \dot{\mathbf{q}}) \dot{\mathbf{q}}+\mathbf{g}(\mathbf{q})=\boldsymbol{\tau}-\mathbf{J}_{h}^{T} \mathbf{W}_{c}
$$

where $\mathbf{q}=\left\{\mathbf{q}_{1}, \ldots, \mathbf{q}_{n}\right\} \in \mathcal{Q}$ is the vector of the generalized configuration variables for the $n$ fingers, with $\mathcal{Q}$ configuration manifold and $\dot{\mathbf{q}} \in T_{q} \mathcal{Q}$ their generalized velocities, belonging to the tangent space of $\mathcal{Q}$ at $\mathbf{q}$. The vector $\tau \in$ $T_{q}^{*} \mathcal{Q}$ represents the generalized actuator forces at the joints, belonging to the co-tangent space of $\mathcal{Q}$ at $\mathbf{q}$. The matrix $\mathbf{J}_{h}$ is the hand Jacobian, that maps the joint velocities to the Cartesian fingertip velocities. From duality, it follows that the transpose $\mathbf{J}_{h}^{T}$ maps the fingertip forces to generalized joint forces. Let $\mathbf{W}_{c}=\left\{\mathbf{W}_{c_{1}}, \ldots, \mathbf{W}_{c_{n}}\right\}$ be the vector of the contact wrenches.

The dynamics of the object are given by:

$$
\mathbf{M}_{0}\left(\mathbf{x}_{0}\right) \ddot{\mathbf{x}}_{0}+\mathbf{C}_{0}\left(\mathbf{x}_{0}, \dot{\mathbf{x}}_{0}\right) \dot{\mathbf{x}}_{0}+\mathbf{g}_{0}\left(\mathbf{x}_{0}\right)=\mathbf{G W}_{c}+\mathbf{F}_{\mathrm{env}}
$$

where $\mathrm{x}_{0} \in \mathcal{X}$ represents the pose of the object, with $\mathcal{X}$ the configuration manifold of the object, and $\dot{\mathrm{x}}_{0}$ its generalized velocity. To avoid singularities due to the local representation of the pose, we can describe the object dynamics globally by applying the Newton-Euler equations to the body configuration expressed in the Special Euclidian group $S E(3)$, and then obtain the Lagrange-D'Alembert representation choosing the local coordinates $\mathrm{x}_{0} \in \mathcal{X}$ for the object configuration. The matrix $\mathbf{G}$ is the grasp matrix, which is a linear map between the contact forces, expressed in the contact frame, and the resultant force on the object, expressed in the object frame [9]. The vector $\mathbf{G} \mathbf{W}_{c}$ describes the effect of the fingertip forces on the object, applied at the contact points. The external forces acting on the object are described by $\mathbf{F}_{\text {env }}$. In the hand and object dynamics, the matrices $\mathbf{M}(\mathbf{q}), \mathbf{M}_{0}\left(\mathbf{x}_{0}\right)$ are the symmetric and positive definite inertia matrices, the matrices $\mathbf{C}(\mathbf{q}, \dot{\mathbf{q}}), \mathbf{C}_{0}\left(\mathbf{x}_{0}, \dot{\mathbf{x}}_{0}\right)$ contain the centrifugal and Coriolis components, and $\mathbf{g}(\mathbf{q})$, $\mathrm{g}_{0}\left(\mathbf{x}_{0}\right)$ are the vectors of generalized gravity forces acting on the hand and the object, respectively [1]. In the context of Lie group theory, the relative configuration of two bodies can be studied using $S E(3)$. The relative instantaneous motion can be studied using the Lie algebra $s e(3)$ associated to $S E(3)$, which is a $6 \mathrm{D}$ algebra, and corresponds to the six possible motions of a rigid body. The wrenches belong to the dual algebra $s e^{*}(3)$. 


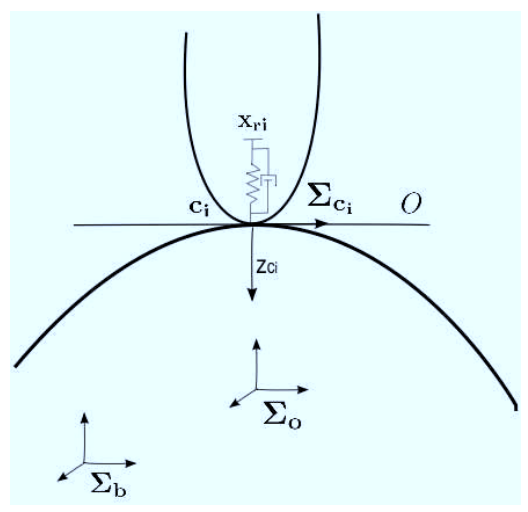

Fig. 2. The geometrical contact model: the soft-pad is modeled as a spring and a damper according to the nonlinear Hunt-Crossley model.

We assume that the fingers have thick compliant layers of viscoelastic material, and the dynamical behavior of the soft layers is modeled as a spring and a damper [10]. The model can be extended to a proper generalization to the full geometrical contact description, as proposed in [11]. In this work, we started from this geometrical analysis of the viscoelastic contacts between two objects without constraints, and extended this to a manipulation context, a complex multibody system including the robotic hand, the soft-pads and the object and subject to constraints. The contact dynamics between the fingers and the object are represented with the same geometrical and energetically consistent model. Moreover, a nonlinear Hunt-Crossley model of the contact is taken into account, for a better physical consistency and description of soft material behavior [12].

In Fig. 2, a schematic representation of the object and one finger in contact is shown. During the contact, a finger with soft-pad is able to transfer to the object four components of the contact wrench $\mathbf{W}_{c_{i}}$, i.e. the three components of the linear contact force and the component of the contact torque around the direction orthogonal to the surface of the object in the point of contact. Our goal is to describe the dynamics of this system in the port-Hamiltonian framework, including the contact dynamics. This allows to present the problem in a more intuitive and compact way. Moreover, given the port-Hamiltonian system representation, an IPC can be easily derived to control the system.

\section{CONTACT MODEL}

In this Section, we derive the dynamic model of the contact, based on the Hunt-Crossley contact model [12]. The Hunt-Crossley model incorporates a spring in parallel with a nonlinear damper to model the viscoelastic dynamics.

In order to obtain a local representation of the Dirac structure of the contact in a matrix form, we have to define a contact frame in the contact point $\mathbf{c}_{i}$ on the object, as indicated in Fig. 2. The contact frame $\boldsymbol{\Sigma}_{c_{i}}$ related to the finger $i$, has the origin in the contact point $\mathbf{c}_{i}$, and the axis $z_{c_{i}}$ is normal to the object surface, pointing inside the object. There is a unique plane $O$ orthogonal to $z_{c_{i}}$ and passing trough $\mathbf{c}_{i}$, spanned by the axis $x_{c_{i}}$ and $y_{c_{i}}$ of the contact frame. In Fig. 2, the object reference frame $\boldsymbol{\Sigma}_{o}$, and the world frame $\boldsymbol{\Sigma}_{b}$ are also depicted.

If we chose a basis of the two screws $\left(\mathbf{r}_{x}, \mathbf{r}_{y}\right)$, representing pure rotations around the two axes $x_{c_{i}}$ and $y_{c_{i}}$ of the contact frame, and a basis of the screws representing rotation around $z_{c_{i}}$ and the three translations $\left(\mathbf{r}_{z}, \mathbf{t}_{x}, \mathbf{t}_{y}, \mathbf{t}_{z}\right)$, we can decompose $s e(3)$ in the direct sum of the two subspaces $S:=$ span $\left\{\mathbf{r}_{z}, \mathbf{t}_{x}, \mathbf{t}_{y}, \mathbf{t}_{z}\right\}$ and $N:=$ span $\left\{\mathbf{r}_{x}, \mathbf{r}_{y}\right\}$, representing the subspace of the transferable wrenches in the contact point and the non transferable wrenches respectively. In particular, the motions in $S$ involve a change in storage of potential energy in the viscoelastic contact [11].

A tangent map $\mathbf{P}$ exists, that projects a twists in $s e(3)$ in the subspace $S$ of motions, and the dual cotangent map $\mathbf{P}^{*}$ :

$$
\mathbf{P}: s e(3) \rightarrow S, \quad \text { and } \quad \mathbf{P}^{*}: S^{*} \rightarrow s e^{*}(3)
$$

Consequently, the elastic storage element, representing the elastic energy stored in the compressed surface of the softpad in contact with the object, is a 4D port with power

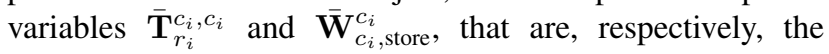
relative twist and the contact elastic wrench expressed in the contact frame and projected in the subspace of motions involving elastic storage of energy. In particular:

$$
\overline{\mathbf{T}}_{r_{i}}^{c_{i}, c_{i}}=\mathbf{P} \mathbf{T}_{r_{i}}^{c_{i}, c_{i}}, \quad \mathbf{W}_{c_{i}, \text { store }}^{c_{i}}=\mathbf{P}^{*} \overline{\mathbf{W}}_{c_{i}, \text { store }}^{c_{i}}
$$

Since the dynamics of the object are dependent of the set of all wrenches acting on it, it is necessary to measure the contact wrenches in order to compute the position of the object center of mass and of the points of contact. Once the measurements of the contact wrenches are available, and the stiffness of the elastic storage element is known, the deformation $(x, y, z, \theta)$ of the soft-pad can be computed in the basis of the screws spanning the subspace of relative motions involving elastic storage of energy $S$.

Writing this deformation, relative to the contact coordinate frame, as an element $\overline{\mathbf{H}} \in S E(3)$ :

$$
\overline{\mathbf{H}}=\left[\begin{array}{cccc}
\cos (\theta) & -\sin (\theta) & 0 & x \\
\sin (\theta) & \cos (\theta) & 0 & y \\
0 & 0 & 1 & z \\
0 & 0 & 0 & 1
\end{array}\right]
$$

the storage of potential energy in the element can be represented by a function $V(\overline{\mathbf{H}}): S E(3) \rightarrow \mathbb{R}$.

If $\overline{\mathbf{H}}(t)$ is known, the relative twist $\mathbf{T}_{r_{i}}^{c_{i}, c_{i}}$ of the object at the contact point with respect to the finger $i$, can be expressed, in theory, in the contact frame as $\mathbf{T}_{r_{i}}^{c_{i}, c_{i}}=\dot{\overline{\mathbf{H}}} \overline{\mathbf{H}}^{-1}$. In practice we can obtain this twist by measuring the deformation of the soft-pad using and estimate the derivative using an observer or by numerical differentiation. The wrench generated due to a deformation $\delta \mathbf{T}_{r_{i}}^{c_{i}, c_{i}}$, related to the relative position of the rigid finger tip $\mathbf{x}_{r_{i}}^{c_{i}}$ and the object contact point $\mathbf{x}_{c_{i}}^{c_{i}}$, has the following expression in the contact frame, 
according to the Hunt-Crossley model ${ }^{1}$ :

$$
\mathbf{W}_{c_{i}}^{c_{i}}=\mathbf{K}_{s_{i}}\left(\delta \mathbf{T}_{r_{i}}^{c_{i}, c_{i}}\right)+\mathbf{D}_{s_{i}}\left(\delta \mathbf{T}_{r_{i}}^{c_{i}, c_{i}}\right) \mathbf{T}_{r_{i}}^{c_{i}, c_{i}}
$$

where $\mathbf{K}_{s_{i}}$ is the two-covariant stiffness tensor [14], such that

$$
\mathbf{K}_{s_{i}}=\mathbf{P}^{*} \overline{\mathbf{K}}_{s_{i}} \mathbf{P}
$$

with $\overline{\mathbf{K}}_{s_{i}}$ the stiffness matrix that relates the 4D port variables $\left(\overline{\mathbf{T}}_{r_{i}}^{c_{i}, c_{i}}, \overline{\mathbf{W}}_{c_{i} \text {,store }}^{c_{i}}\right)$, and $\mathbf{D}_{s_{i}}\left(\delta \mathbf{T}_{r_{i}}^{c_{i}, c_{i}}\right)$ is the damping matrix that depends on the deformation, and is defined locally as $\left(\delta \mathbf{T}_{r_{i}}^{c_{i}, c_{i}}\right)^{T} \mathbf{D}$, with $\mathbf{D}$ a constant diagonal matrix depending on the structure and on the material of the softpad. By considering the map $\mathbf{P}^{*}$, we can express the vector of contact wrenches with respect to the contact deformation:

$$
\mathbf{W}_{c_{i}}^{c_{i}}=\mathbf{P}^{*}\left(\overline{\mathbf{K}}_{s_{i}}\left(\delta \overline{\mathbf{T}}_{r_{i}}^{c_{i}, c_{i}}\right)+\overline{\mathbf{D}}_{s_{i}}\left(\delta \overline{\mathbf{T}}_{r_{i}}^{c_{i}, c_{i}}\right) \overline{\mathbf{T}}_{r_{i}}^{c_{i}, c_{i}}\right)
$$

where $\overline{\mathbf{D}}_{s_{i}}$ is defined analogous to $\mathbf{D}_{s_{i}}$, but with respect to the $4 \mathrm{D}$ vector of the deformations:

$$
\delta \overline{\mathbf{T}}_{r_{i}}^{c_{i}, c_{i}}=[x, y, z, \theta]^{T}
$$

which represents the deformation of the soft-pad in the allowed directions.

\section{PORT-HAMILTONIAN MODEL}

In this Section, we intend to derive the port-Hamiltonian equations of the complete system. Therefore, starting from the general representation of Fig. 1, we can characterize the Dirac structure by explicitly describing the three different sub-systems, i.e. the hand, the object and the contact. The complete system is composed by a number of storage and dissipative elements and interaction ports, connected together, as shown in Fig. 3.

The power port between the Dirac structure of the fingers and the Dirac structure of the contact is a multi-bond port. In particular, the interconnection between the hand and the contact is identified by the effort $\mathbf{W}_{c_{f}}^{b}$, i.e. the vector of the wrenches exerted by the soft-pads (due to the object) on the fingers, and by the flow $\mathbf{T}_{r}^{b}=\mathbf{J}_{h} \dot{\mathbf{q}} \in \mathbb{R}^{6 n}$, i.e. the vector of the twists of the (rigid) finger tip in the base frame.

The power port between the Dirac structure of the object and the Dirac structure of the contact is a multi-bond port as well. The port is characterized by the effort $\mathbf{W}_{c_{o}}^{b}$, i.e. the vector of wrenches exerted by the fingers on the object, and by the flow $\mathbf{T}_{c}^{b}=\mathbf{G}^{T}\left(\mathbf{x}_{0}\right) \dot{\mathbf{x}}_{0} \in \mathbb{R}^{6 n}$, i.e. the vector of the twists of the object in the contact points in the base frame. The power port between the storage element of the soft-pads and the Dirac structure of the contact is characterized by the effort $\overline{\mathbf{W}}_{c \text {,store }}^{c}$, i.e. the vector of the wrench transferable component due to the (soft-pad) springs, represented by the vector of the partial derivatives of the total Hamiltonian energy function with respect to the vector of the states of the (soft-pad) springs, $\frac{\partial H}{\partial \mathbf{s}_{c}^{T}}$, and by the flow $\overline{\mathbf{T}}_{r}^{c, c}=\dot{\mathbf{s}}_{c}$, i.e. the vector of the relative twists between the fingers and the object, projected in the subspace $S$ of

\footnotetext{
${ }^{1}$ In the Hunt-Crossley model, the stiffness and damping forces are taken proportional to $\delta^{m}$, with $\delta$ the scalar deformation and $m$ usually close to unity. For simplicity, we have assumed $m=1$ in this work.
}

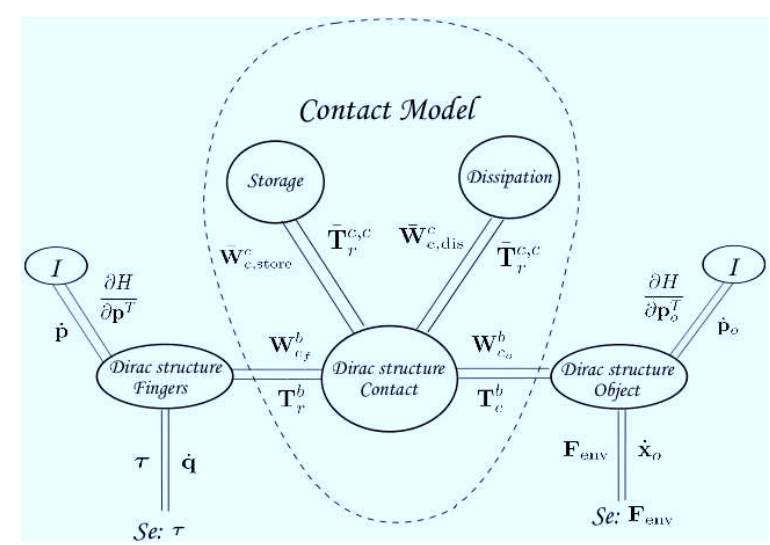

Fig. 3. Dirac structure of the whole constrained port-Hamiltonian system. The fingers and the object are connected with two effort sources, that are, respectively, the control action and the external forces acting on the object.

the motion involving elastic storage of energy. The power port between the dissipative element of the soft-pads and the Dirac structure of the contact is characterized by the effort $\overline{\mathbf{W}}_{c \text {,dis }}^{c}$, i.e. the vector of the wrench transferable component due to the (soft-pad) dampers, and by the flow $\overline{\mathbf{T}}_{r}^{c, c}$.

To complete the description of the system represented in Fig. 3, we need to consider the inertia of the fingers, connected to the finger Dirac structure by means of the power flow defined by the conjugate variables $\left(\dot{\mathbf{p}}, \frac{\partial H}{\partial \mathbf{p}^{T}}\right)$, and the control port described by $(\dot{\mathbf{q}}, \boldsymbol{\tau})$. For the object, we need to consider the inertia of the object, connected to the object Dirac structure by means of the power flow defined by the conjugate variables $\left(\dot{\mathbf{p}}_{o}, \frac{\partial H}{\partial \mathbf{p}_{o}^{T}}\right)$, and the environment interaction port described by $\left(\dot{\mathbf{x}}_{o}, \mathbf{F}_{\text {env }}\right)$. For the sake of simplicity, we neglect Coriolis effects. This simplification is justified because of the small work space we consider, and the relatively low velocities of the system.

For clarity, in the figure we have omitted the power port of the gravity external force, that we take in account in the port-Hamiltonian equations, for the fingers and the object, i.e. $\left(\dot{\mathbf{q}}_{w}, \frac{\partial H}{\partial \mathbf{q}^{T}}\right)$ and $\left(\dot{\mathbf{x}}_{o, w}, \frac{\partial H}{\partial \mathbf{x}_{o}^{T}}\right)$ respectively.

\section{A. Dirac Structure of the fingers}

The Hamiltonian energy function of the fingers is

$$
H\left(\mathbf{s}_{c}, \mathbf{p}, \mathbf{q}\right)=\frac{1}{2} \mathbf{p}^{T} \mathbf{M}^{-1}(\mathbf{q}) \mathbf{p}+\frac{1}{2} \mathbf{s}_{c}^{T} \overline{\mathbf{K}}_{s} \mathbf{s}_{c}+V(\mathbf{q})
$$

where the first term is the kinetic energy of the finger, the second is the potential energy of the springs that model the soft-pads and $V(\mathbf{q})$ is the potential energy of the fingers due to gravity. The Hamiltonian equations of the fingers are:

$$
\dot{\mathbf{p}}=-\frac{\partial H}{\partial \mathbf{q}^{T}}+\mathbf{J}_{h}^{T} \mathbf{W}_{c_{f}}^{b}+\boldsymbol{\tau}, \quad \dot{\mathbf{q}}=\frac{\partial H}{\partial \mathbf{p}^{T}}
$$

The Dirac structure can be represented by a skew- 
symmetric matrix:

$$
\left[\begin{array}{c}
\dot{\mathbf{p}} \\
\dot{\mathbf{q}} \\
\mathbf{T}_{r}^{b} \\
\dot{\mathbf{q}} w
\end{array}\right]=\left[\begin{array}{cccc}
\mathbf{0} & -\mathbf{1} & -\mathbf{J}_{h}^{T} & -\mathbf{1} \\
\mathbf{1} & \mathbf{0} & \mathbf{0} & \mathbf{0} \\
\mathbf{J}_{h} & \mathbf{0} & \mathbf{0} & \mathbf{0} \\
\mathbf{1} & \mathbf{0} & \mathbf{0} & \mathbf{0}
\end{array}\right]\left[\begin{array}{c}
\frac{\partial H}{\partial \mathbf{p}^{T}} \\
-\boldsymbol{\tau} \\
-\mathbf{W}_{c_{f}}^{b} \\
\frac{\partial H}{\partial \mathbf{q}^{T}}
\end{array}\right]
$$

where $\mathbf{0}$ and $\mathbf{1}$ denote zero and identity matrices of appropriate dimensions.

\section{B. Dirac Structure of the object}

The Hamiltonian energy function of the object is given by

$$
H\left(\mathbf{p}, \mathbf{x}_{o}\right)=\frac{1}{2} \mathbf{p}_{o}^{T} \mathbf{M}^{-1} \mathbf{p}_{o}+V_{o}\left(\mathbf{x}_{o}\right)
$$

where $V_{o}\left(\mathbf{x}_{o}\right)$ is the potential energy of the object due to gravity. The Hamiltonian equations of the object are:

$$
\dot{\mathbf{p}}_{o}=-\frac{\partial H}{\partial \mathbf{x}_{o}^{T}}+\mathbf{G} \mathbf{W}_{c_{o}}^{b}+\mathbf{F}_{\mathrm{env}}, \quad \dot{\mathbf{x}}_{o}=\frac{\partial H}{\partial \mathbf{p}_{o}^{T}}
$$

The Dirac structure can be represented by a skewsymmetric matrix:

$$
\left[\begin{array}{c}
\dot{\mathbf{p}}_{o} \\
\dot{\mathbf{q}}_{o} \\
\mathbf{T}_{c}^{b} \\
\dot{\mathbf{x}}_{o, w}
\end{array}\right]=\left[\begin{array}{cccc}
\mathbf{0} & -\mathbf{1} & -\mathbf{G} & -\mathbf{1} \\
\mathbf{1} & \mathbf{0} & \mathbf{0} & \mathbf{0} \\
\mathbf{G}^{T} & \mathbf{0} & \mathbf{0} & \mathbf{0} \\
\mathbf{1} & \mathbf{0} & \mathbf{0} & \mathbf{0}
\end{array}\right]\left[\begin{array}{c}
\frac{\partial H}{\partial \mathbf{p}_{o}^{T}} \\
-\mathbf{F}_{\mathrm{env}} \\
-\mathbf{W}_{c_{o}}^{b} \\
\frac{\partial H^{\prime}}{\partial \mathbf{x}_{o}^{T}}
\end{array}\right]
$$

\section{Dirac Structure of the contact}

In order to obtain a local representation of the Dirac structure of the contact in a matrix form, we have to choose a reference frame of the contact, and consider the transformation between the contact frame $\boldsymbol{\Sigma}_{c}$ and the world frame $\Sigma_{b}$. In the case of soft finger contact model, the Dirac structure of the contact is the following:

$$
\left[\begin{array}{c}
\mathbf{W}_{c_{f}}^{b} \\
\mathbf{W}_{c_{o}}^{b} \\
\overline{\mathbf{T}}_{r}^{c, c} \\
\dot{\mathbf{s}}_{c}
\end{array}\right]=\left[\begin{array}{cccc}
\mathbf{0} & \mathbf{0} & -\mathbf{A}^{*} & -\mathbf{A}^{*} \\
\mathbf{0} & \mathbf{0} & \mathbf{A}^{*} & \mathbf{A}^{*} \\
\mathbf{A} & -\mathbf{A} & \mathbf{0} & \mathbf{0} \\
\mathbf{A} & -\mathbf{A} & \mathbf{0} & \mathbf{0}
\end{array}\right]\left[\begin{array}{c}
\mathbf{T}_{r}^{b} \\
\mathbf{T}_{c}^{b} \\
\overline{\mathbf{W}}_{c, \text { dis }}^{c} \\
\frac{\partial H_{o}}{\partial \mathbf{s}_{c}^{T}}
\end{array}\right]
$$

where $\mathbf{A}^{*}$ is the matrix representation of the operator:

$$
\mathbf{A}^{*}:=\operatorname{diag}\left\{\mathbf{A}_{1}^{*}, \ldots, \mathbf{A}_{n}^{*}\right\}, \quad \mathbf{A}_{i}^{*}=\left(s_{\Delta, i}-1\right) \operatorname{Ad}_{\mathbf{H}_{b}^{c_{i}}}^{T} \mathbf{P}^{*}
$$

The matrix $\mathbf{A}$ is the dual operator to $\mathbf{A}^{*}$, and is expressed as the transpose of matrix expression for $\mathbf{A}^{*}$. The binary signal $s_{\Delta, i}$ is defined as $s_{\Delta, i}=1$ if there is no contact, and $s_{\Delta, i}=0$ if there is contact [11]. If the position of the object is unknown, as in an unstructured environment, the binary signal can be related to the signal of a force sensor located in the soft-pad.

The adjoint operator $\operatorname{Ad}_{\mathbf{H}_{b}^{c_{i}}}$ transforms the relative twist between the rigid part of the finger $i$ and the contact point on the object from coordinates in $\boldsymbol{\Sigma}_{b}$, the world frame, to $\Sigma_{c_{i}}$, the contact coordinate frame. The first two equations give the expression of the contact forces acting on the finger and on the object in case of contact and non-contact, thanks to the binary signal. In case of non-contact they are zero and in the other case they are obviously opposite, and they have the expression of Hunt-Crossley model.

\section{Dirac Structure of whole system}

In this Section, we derive the Dirac structure of the whole system by combining the three Dirac structures derived for the different sub-systems. It follows that the skew-symmetric Dirac matrix, from Eq. (2) at the top of next page, describes the energy flows through the system.

From Eq. (2) it is possible to obtain the input-state-output port-Hamiltonian system of the whole system. The flow and effort variables of control and interaction port are split into conjugated input-output pairs. The general form is the following:

$$
\begin{aligned}
& \dot{\mathbf{x}}=(\mathbf{J}(\mathbf{x})-\mathbf{R}(\mathbf{x})) \frac{\partial H}{\partial \mathbf{x}^{T}}+\mathbf{g}(\mathbf{x}) \mathbf{u} \\
& \mathbf{y}=\mathbf{g}^{T}(\mathbf{x}) \frac{\partial H}{\partial \mathbf{x}^{T}}
\end{aligned}
$$

where $(\mathbf{u}, \mathbf{y})$ are the input/output pairs corresponding to the control port, the matrix $\mathbf{J}(\mathbf{x})$ is a skew-symmetric matrix representing the port topology defined by the Dirac structure, while the matrix $\mathbf{R}(\mathbf{x})=\mathbf{R}^{T}(\mathbf{x}) \geq 0$ specifies the energy dissipation. In our case we have the state $\mathbf{x}=$ $\left[\begin{array}{lllll}\mathbf{p} & \mathbf{q} & \mathbf{p}_{o} & \mathbf{x}_{o} & \mathbf{s}_{c}\end{array}\right]^{T}$ and energy function

$$
\begin{aligned}
H(\mathbf{x})= & \frac{1}{2} \mathbf{p}^{T} \mathbf{M}^{-1}(\mathbf{q}) \mathbf{p}+\frac{1}{2} \mathbf{s}_{c}^{T} \overline{\mathbf{K}}_{s} \mathbf{s}_{c}+V(\mathbf{q}) \\
& +\frac{1}{2} \mathbf{p}_{o}^{T} \mathbf{M}^{-1} \mathbf{p}_{o}+V_{o}\left(\mathbf{x}_{o}\right)
\end{aligned}
$$

Furthermore, we obtain:

$$
\begin{aligned}
& \mathbf{J}(\mathbf{x})= {\left[\begin{array}{ccccc}
\mathbf{0} & -\mathbf{1} & \mathbf{0} & \mathbf{0} & -\mathbf{J}_{h}^{T} \mathbf{A} \\
\mathbf{1} & \mathbf{0} & \mathbf{0} & \mathbf{0} & \mathbf{0} \\
\mathbf{0} & \mathbf{0} & \mathbf{0} & -\mathbf{1} & \mathbf{G} \mathbf{A} \\
\mathbf{0} & \mathbf{0} & \mathbf{1} & \mathbf{0} & \mathbf{0} \\
\mathbf{A}^{T} \mathbf{J}_{h} & \mathbf{0} & -\mathbf{A}^{T} \mathbf{G}^{T} & \mathbf{0} & \mathbf{0}
\end{array}\right] } \\
& \mathbf{R}(\mathbf{x})= {\left[\begin{array}{cccccc}
\mathbf{J}_{h}^{T} \mathbf{A} \overline{\mathbf{D}} \mathbf{A}^{T} \mathbf{J}_{h} & \mathbf{0} & \mathbf{J}_{h}^{T} \mathbf{A} \overline{\mathbf{D}} \mathbf{A}^{T} \mathbf{G}^{T} & \mathbf{0} & \mathbf{0} \\
\mathbf{0} & \mathbf{0} & \mathbf{0} & \mathbf{0} & \mathbf{0} \\
-\mathbf{G} \mathbf{A} \overline{\mathbf{D}} \mathbf{A}^{T} \mathbf{J}_{h} & \mathbf{0} & \mathbf{G} \mathbf{A} \overline{\mathbf{D}} \mathbf{A}^{T} \mathbf{G}^{T} & \mathbf{0} & \mathbf{0} \\
\mathbf{0} & \mathbf{0} & \mathbf{0} & \mathbf{0} & \mathbf{0} \\
\mathbf{0} & \mathbf{0} & \mathbf{0} & \mathbf{0} & \mathbf{0}
\end{array}\right] } \\
& \mathbf{g}(\mathbf{x})=\left[\begin{array}{lllll}
\mathbf{0} & \mathbf{1} & \mathbf{0} & \mathbf{0} & \mathbf{0} \\
\mathbf{0} & \mathbf{0} & \mathbf{0} & \mathbf{1} & \mathbf{0}
\end{array}\right]^{T} \\
& \mathbf{u}=\left[\begin{array}{llll}
\boldsymbol{\tau} & \mathbf{F}_{\mathrm{env}}
\end{array}\right]^{T}, \quad \mathbf{y}=\left[\begin{array}{ll}
\dot{\mathbf{q}} & \dot{\mathbf{x}}_{o}
\end{array}\right]^{T}
\end{aligned}
$$

The matrix $\mathbf{J}(\mathbf{x})$ is obtained from Eq. (2) by inspection, as well as matrix $\mathbf{g}(x)$. The matrix $\mathbf{R}(\mathbf{x})$ is obtained considering the expression of the damping force in Eq. (1).

\section{SIMULATIONS}

For the validation of the model, a simple example modeled in 20-sim simulation software [15] is considered. We implement the bond graph representation of two fingers with soft-pads, that interacts with an object in a plane. An IPC is implemented to control the joint torques:

$$
\boldsymbol{\tau}=\mathbf{g}(\mathbf{q})+\mathbf{J}_{h}^{T}\left[\mathbf{K}_{c}\left(\mathbf{x}_{r_{d}}-\mathbf{x}_{r}\right)\right]-\mathbf{D}_{c} \dot{\mathbf{q}}
$$

where the terms $\mathbf{K}_{c}$ and $\mathbf{D}_{c}$ are the proportional and derivative control gains. Here the desired rigid finger tips positions 


\begin{tabular}{|c|c|c|c|c|c|c|c|c|c|c|}
\hline$\dot{\mathbf{p}}$ & & 0 & -1 & -1 & 0 & 0 & 0 & $-\mathbf{J}_{h}^{T} \mathbf{A}$ & $-\mathbf{J}_{h}^{T} \mathbf{A}^{-}$ & $-\tau$ \\
\hline$\dot{\mathbf{q}}$ & & 1 & 0 & 0 & 0 & 0 & 0 & 0 & 0 & $\partial H$ \\
\hline$\dot{\mathbf{q}}_{w}$ & & 1 & 0 & 0 & 0 & 0 & 0 & 0 & 0 & \\
\hline$\dot{\mathbf{p}}_{o}$ & $=$ & 0 & 0 & 0 & 0 & -1 & -1 & GA & GA & $\frac{\partial H}{\partial \mathbf{p}_{o}^{T}}$ \\
\hline$\dot{\mathbf{x}}_{o}$ & - & $\mathbf{0}$ & 0 & 0 & 1 & 0 & 0 & $\mathbf{0}$ & $\mathbf{0}$ & $-\mathbf{F}_{\mathrm{env}}$ \\
\hline$\dot{\mathbf{x}}_{o, w}$ & & $\mathbf{0}$ & 0 & 0 & 1 & 0 & 0 & 0 & $\mathbf{0}$ & $\partial H$ \\
\hline $\mathbf{T}_{r}^{c, c}$ & & $\mathbf{A}^{T} \mathbf{J}_{h}$ & 0 & 0 & $-\mathbf{A}^{T} \mathbf{G}^{T}$ & 0 & 0 & 0 & 0 & $\begin{array}{l}\partial \mathbf{x}_{o}^{T} \\
\bar{y}\end{array}$ \\
\hline$\dot{\mathbf{s}}_{c}$ & & $\mathbf{A}^{T} \mathbf{J}_{h}$ & 0 & 0 & $-\mathbf{A}^{T} \mathbf{G}^{T}$ & $\mathbf{0}$ & 0 & 0 & 0 & {$\left[\begin{array}{c}\mathbf{W}_{c, \text { dis }} \\
\frac{\partial H}{\partial \mathbf{s}_{c}^{T}}\end{array}\right.$} \\
\hline
\end{tabular}

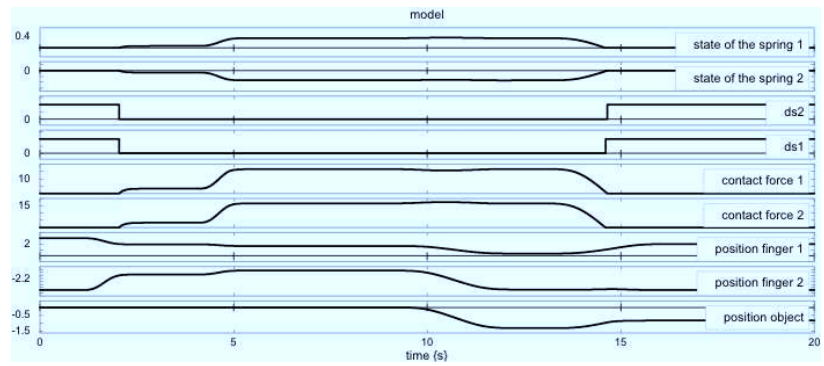

Fig. 4. Simulation results - The simulations validate the model with control of the internal force and control of the object motion. When the binary signal switches from 1 to 0 , the fingers are in contact with the object.

$\mathbf{x}_{r_{d}}$ are derived considering the desired forces at the contact and the model of the soft-pad. By means of the virtual position, we are able to regulate the motion of the object and, through the null space of the grasp matrix, to regulate the internal forces as well by controlling the relative position of the finger tips. The soft-pad is modeled as a spring and a damper, with a stiffness constant $k=50 \mathrm{~N} / \mathrm{m}$, and damping constant $d=3 \mathrm{Ns} / \mathrm{m}$. When the soft-pad touches the object, the binary signal $s_{\Delta}$ switches from 1 to 0 .

The simulation is divided in three phases. First, the fingers move, touch the object and push against it until the internal forces are regulated at $16.5 \mathrm{~N}$. Then, the fingers move the object, while maintaining the regulation of the internal forces at the same value. The difference from the contact force of the two fingers are due to the inertia of the fingers and of the object. In the third step the fingers move away from the object. The object continues to move in the left direction, due to the deformation in the soft-pads, and it will stop due to the friction. Fig. 4 shows the diagrams of the positions of the finger tips and of the object, and the contact forces at the finger tips. For a better understanding of the simulation results, a video of a 3D animation in 20-sim has been made and attached to the paper.

\section{CONCLUSIONS AND FUTURE WORK}

In this paper, we presented a port-Hamiltonian analysis and model of a multi-fingered robotic hand with softpads while grasping and manipulating an object. The main advantage of this formalism is that it allows to describe the behavior of the system in terms of energy storage and energy flow, using the concept of power ports. This formalism represents an efficient and useful way to describe the interaction between the object and the fingers, as well as the interaction of the whole system with the environment.

Using screw theory and Lie group theory, the viscoelastic description of the contact can be expressed as a power continuous interconnection, represented by a Dirac structure. Therefore, we are able to describe non-contact to contact transition, and contact viscoelasticity without changing the dynamical equations of the model.

Future work will focus on the passive control of the internal and external forces, also in case of sharing tasks with a human being. Moreover, different models of the softpad will be used, like soft-pads with nonlinear stiffness [13].

\section{REFERENCES}

[1] R.M. Murray, Z. Li and S.S. Sastry, A Mathematical Introduction to Robot Manipulation, CRC Press, 1994.

[2] P.C. Breedveld, Physical Systems Theory in Terms of Bond Graphs, PhD thesis, University of Twente, Enschede, 1984.

[3] S. Stramigioli, Modeling and IPC Control of Interactive Mechanical Systems: A Coordinate-free Approach, Springer-Verlag, 2001.

[4] N. Hogan, Impedance control: An approach to manipulation: Parts IIII, ASME Trans. Jour. Dynamic Systems and Measurement Control, vol. 107, pp.1-24, 1985.

[5] S. Stramigioli and V. Duindam, Variable Spatial Springs for Robot Control, IEEE/RSJ Int. Conf. on Intelligent Robots and Systems, 2001.

[6] A.J. van der Schaft and B.M. Maschke, On the Hamiltonian formulation of nonholonomic mechanical systems, Reports on Mathematical Physics, vol. 34, no. 2, pp. 225-233, 1994.

[7] A.J. van der Schaft, $L_{2}$-Gain and Passivity Techniques in Nonlinear Control, Springer, 2000.

[8] S. Stramigioli and V. Duindam, Port Based Modeling of Spatial ViscoElastic Contacts, European Journal of Control, pp. 510-519, 2004.

[9] B. Siciliano and O. Khatib (Eds.), Springer Handbook of Robotics, Chapter 28 Grasping, Springer-Verlag, 2008.

[10] L. Biagiotti, P. Tiezzi, C. Melchorri and G. Vassura, Modelling and controlling the compliance of a robotic hand with soft finger-pads, IEEE Int. Conf. on Robotics and Automation, 2004.

[11] S. Stramigioli and V. Duindam, Modeling the kinematics and dynamics of compliant contact, IEEE Int. Conf. on Robotics and Automation, 2003.

[12] N. Diolaiti, C. Melchorri and S. Stramigioli, Contact Impedance Estimation for Robotic Systems, IEEE Trans. on Robotics, vol. 21, pp. 925-935, 2005.

[13] G. Berselli and G. Vassura, Differentiated layer design to modify the compliance of soft-pads for robotics limbs, IEEE Int. Conf. on Robotics and Automation, 2009.

[14] M. Zefran and V. Kumar, Affine connections for the Cartesian stiffness matrix, IEEE Int. Conf. on Robotics and Automation, 1997.

[15] Controllab Products B.V., 20-sim, http://www.20sim.com, 2009. 